Rochester Institute of Technology

RIT Scholar Works

5-16-2018

\title{
Storage and Retrieval of Optical Information in Levitated Cavityless Optomechanics
}

\author{
Pardeep Kumar \\ Rochester Institute of Technology \\ Mishkatul Bhattacharya \\ Rochester Institute of Technology
}

Follow this and additional works at: https://scholarworks.rit.edu/other

\section{Recommended Citation}

Pardeep Kumar, M. Bhattacharya, "Storage and retrieval of optical information in levitated cavityless optomechanics," Proc. SPIE 10660, Quantum Information Science, Sensing, and Computation X, 106600K (16 May 2018); doi: 10.1117/12.2304645

This Conference Paper is brought to you for free and open access by the Faculty \& Staff Scholarship at RIT Scholar Works. It has been accepted for inclusion in Presentations and other scholarship by an authorized administrator of RIT Scholar Works. For more information, please contact ritscholarworks@rit.edu. 


\section{Storage and retrieval of optical information in levitated cavityless optomechanics}

Pardeep Kumar, M. Bhattacharya

Pardeep Kumar, M. Bhattacharya, "Storage and retrieval of optical information in levitated cavityless optomechanics," Proc. SPIE 10660, Quantum Information Science, Sensing, and Computation X, 106600K (16 May 2018); doi: 10.1117/12.2304645

SPIE Event: SPIE Commercial + Scientific Sensing and Imaging, 2018, Orlando, Florida, United States 


\title{
Storage and Retrieval of Optical Information in Levitated Cavityless Optomechanics
}

\author{
Pardeep Kumar and M. Bhattacharya* \\ School of Physics and Astronomy, Rochester Institute of Technology, 84 Lomb Memorial \\ Drive, Rochester, NY 14623, USA
}

\begin{abstract}
We theoretically consider light storage in a single nanoparticle levitated in an optical dipole trap and subjected to nonlinear feedback cooling. The storage protocol is realized by controlling the coupling between mechanical displacement and signal pulse by maneuvering the intensity of writing and readout pulses. The process involves writing and readout pulses at one mechanical frequency below the signal pulse. We demonstrate that during the writing pulse, a signal pulse is stored as a mechanical excitation of the nanoparticle oscillation. It is then shown that a readout pulse at later time can retrieve the stored optical information from the mechanical oscillator. A long storage lifetime of $2 \mathrm{~ms}$ is obtained in our system due to the absence of clamping losses. Further, we describe that our protocol can be used for wavelength conversion and shows a saturation in the conversion efficiency as a function of cooperativities of the writing and readout pulses. We also illustrate that the presence of linear feedback heating can lead to the amplification of the retrieved photon energy. Our prototype for light storage with levitated optomechanics can be used to explore the possibility of quantum memories for photonic states.
\end{abstract}

Keywords: Storage, Retrieval, Conversion Efficiency, Levitation

\section{INTRODUCTION}

Optical information storage and retrieval is a key ingredient for long-distance quantum communication. ${ }^{1}$ In the last decade, various techniques for light storage have been realized in atomic ensembles ${ }^{2-5}$ spin systems ${ }^{6,7}$ and solid-state media. ${ }^{8,9}$ Recently, cavity optomechanical oscillators ${ }^{10}$ in which optical fields couple to mechanical oscillations via radiation pressure force, have provided a versatile platform for storing optical information. ${ }^{11,12}$ Compared to atomic or spin systems, optomechanical systems allow for light stored as a mechanical excitation at a given wavelength to be retrieved at a later time at any desired wavelength ${ }^{13-16}$ thereby providing an unique platform for hybrid quantum networks. Apart from light storage, the optomechanical interactions have also led to the realization of the cooling of mechanical mode to its ground state, ${ }^{17,18}$ strong coupling between optical and mechanical modes ${ }^{19}$ and optomechanically-induced transparency. ${ }^{20-22}$

Significantly, the experimental realizations in cavity-based optomechanical oscillators suffer from heating and decoherence produced by the clamping losses. Also, the use of optical cavities places restrictions on the optical wavelengths, as they need to be resonant. These restrictions can be circumvented by means of levitated cavityless optomechanics which does not use mechanical clamping or optical resonators. The levitation of the mechanical oscillator can be provided by using optical ${ }^{23,24}$ or magnetic fields. ${ }^{25,26}$ In particular, optically levitated nanoparticles have been proposed for ground-state cooling, ${ }^{27-29}$ preparation of quantum superposition states $^{30}$ and ultra-sensitive applications. ${ }^{31-33}$

In this paper, we devise an optical memory protocol using a single nanoparticle levitated in an optical dipole trap subjected to nonlinear feedback. The protocol works by controlling the coupling between mechanical displacement and signal field by means of writing and reading pulses which are at one mechanical frequency below the signal field. In the presence of writing pulse, the signal pulse interacts with the nanoparticle and is stored as a mechanical excitation. The readout pulse at a later time retrieves the optical information from the mechanical excitation. We demonstrate that the storage time of the optical memory is determined by the relatively long decay time of the mechanical oscillator. The absence of clamping losses in levitated systems provides a storage

E-mail: mxbsps@rit.edu

Quantum Information Science, Sensing, and Computation X, edited by Eric Donkor, Michael Hayduk, Proc. of SPIE Vol. 10660, 106600K · (c) 2018 SPIE CCC code: $0277-786 X / 18 / \$ 18 \cdot$ doi: $10.1117 / 12.2304645$ 
time of 2 ms which is one thousand times longer than reported in cavity based optomechanical light storage. ${ }^{11}$ We further describe the wavelength conversion in which the input signal at one wavelength is converted into retrieved photons at another wavelength and the energy conversion efficiency obtained shows a saturation which quantifies the impedance matching. ${ }^{16}$ Furthermore, the mechanical oscillations of the levitated nanoparticle can be damped or amplified by means of feedback mechanism. ${ }^{34}$ In the present work, by applying linear feedback heating, ${ }^{35}$ we achieve amplification of the retrieved photon energy.

\section{MODEL}

We present an optical memory protocol using a single nanoparticle of mass $m$ which is trapped in vacuum by a focused Gaussian beam resulting in harmonic mechanical oscillations, as shown in Fig. 1. For small amplitudes, the oscillations along three spatial directions are uncoupled and may be considered independently. Here, we consider the oscillations of the nanoparticle in the dipole trap along the $\mathrm{x}$-axis. The position of the nanoparticle is monitored continuously by interferometric techniques. ${ }^{24,34}$ Further, a feedback mechanism is used to cause additional damping of the nanoparticle thereby giving rise to cooling, in addition to some backaction heating. To implement this model as an optical memory, we use writing and readout pulses one mechanical frequency below the signal pulse. The writing and signal pulses arrive simultaneously, followed later by readout pulse, as shown in Fig. 1. The writing pulse controls the coupling between signal pulse and mechanical displacement thereby converting the signal pulse into a mechanical excitation via the optomechanical coupling. The reading pulse at a later time converts the mechanical excitation back into an optical pulse at a desired wavelength.

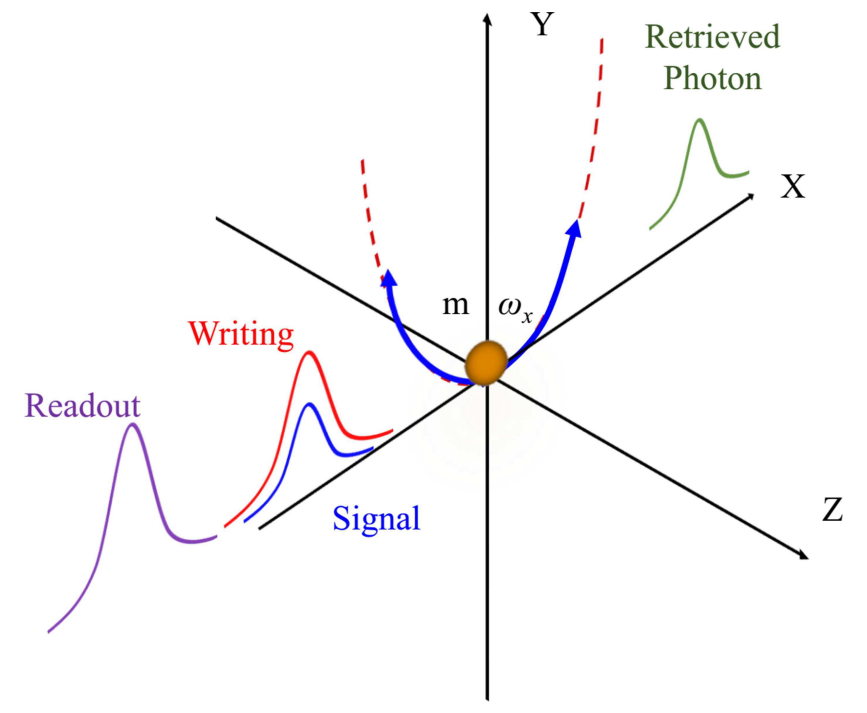

Figure 1. Schematic of light storage and retrieval with a levitated nanoparticle of mass $m$ trapped in an optical dipole trap and oscillating with frequency $\omega_{x}$. The particle is subjected to nonlinear feedback cooling. ${ }^{29} \mathrm{~A}$ writing and signal pulse interact simultaneously with the nanoparticle thereby causing the storage of the signal as a mechanical excitation. The readout pulse at a later time results in the retrieval of photons from the mechanical excitation.

The dynamics of this levitated optomechanical system is described by the following master equation ${ }^{29,36}$

$\dot{\rho}=\frac{1}{i \hbar}[H, \rho]-\left(\frac{\gamma_{\mathrm{opt}}+D_{p}}{2}\right) \mathcal{D}[Q] \rho-\frac{D_{q}}{2} \mathcal{D}[P] \rho-i \frac{\gamma_{g}}{2}[Q,\{P, \rho\}]-i \gamma_{f}\left[Q^{3},\{P, \rho\}\right]-\Gamma_{f} \mathcal{D}\left[Q^{3}\right] \rho+\mathcal{B D}[a] \rho$,

where, the first term on the right-hand side of the above equation represents the unitary evolution of the optomechanical system described by the Hamiltonian $H=H_{0}+H_{\text {int }}$, where $H_{0}$ is the bare Hamiltonian of the mechanical oscillator and optical field and $H_{\text {int }}$ is the optomechanical coupling Hamiltonian between mechanical 
oscillator and the optical field, respectively. The second (third) term describes the momentum (position) diffusion of the nanoparticle due to collisions with background gas, with momentum (position) diffusion coefficient $D_{p}=$ $2 \eta_{f} k_{B} T x_{0}^{2} / \hbar^{2}\left(D_{q}=\eta_{f} \hbar^{2} / 24 k_{B} T m^{2} x_{0}^{2}\right)$, where, $T$ is the gas temperature, $k_{B}$ is Boltzmann's constant and $x_{0}=\sqrt{\hbar / 2 m \omega_{x}}$ is the zero point fluctuation of the mechanical oscillator. Also, $\gamma_{\text {opt }}=\mathcal{A}_{t}+\mathcal{A}_{w}+\mathcal{A}_{s}+\mathcal{A}_{r}$ represents the heating rate due to trap, writing, signal and readout photon scattering, respectively. The fourth term accounts for gas damping at a rate $\gamma_{g}=\eta_{f} / 2 m$, where coefficient of friction $\eta_{f}=6 \pi \mu R$, $\mu$ is the dynamic viscosity of the surrounding gas and $R$ is the radius of nanoparticle. The fifth and sixth terms represent the nonlinear feedback damping and the corresponding feedback backaction, respectively. The feedback drift and diffusion are characterized by the coefficients $\gamma_{f}=\chi^{2} \Phi G$ and $\Gamma_{f}=\chi^{2} \Phi G^{2}$, respectively, where $\chi$ is the scaled optomechanical coupling, $\Phi$ is the average detected photon flux and $G$ is the feedback gain. The last term in above equation represents the optical damping of the writing, signal and readout pulse and is characterized by $\mathcal{B}=\mathcal{B}_{w}+\mathcal{B}_{s}+\mathcal{B}_{r}$. The respective dimensionless position and momentum operators are $Q=b^{\dagger}+b$ and $P=i\left(b^{\dagger}-b\right)$. The creation and annihilation operators of the optical field (mechanical oscillator) are $a^{\dagger}\left(b^{\dagger}\right)$ and $a(b)$. The Lindblad superoperator in Eq. (1) is written as $\mathcal{D}[Q] \rho=Q^{\dagger} Q \rho+\rho Q^{\dagger} Q-2 Q \rho Q^{\dagger}$.

Now the interaction Hamiltonian between mechanical oscillator and the optical field is written as

$$
H_{\text {int }}=-\hbar g a^{\dagger} a\left(b^{\dagger}+b\right),
$$

where, $g=V \frac{2 \epsilon_{c} \omega_{s} \Delta \omega_{s} x_{0}}{\pi^{2} w_{0}^{2} c} \frac{\Delta x}{w_{0}^{2}}$ is the optomechanical coupling constant. Here, $V$ is the volume of the nanoparticle, $\epsilon_{c}$ is the effective relative permittivity of the dielectric, $\omega_{s}$ is the frequency of the applied signal field, $\Delta \omega_{s}$ is the signal laser linewidth, $w_{0}$ is the waist of the signal. We also have assumed that signal beam is shifted from trap by a small amount $\Delta x$. The Hamiltonian in Eq. (2) can be linearized by using

$$
a=\sqrt{n_{i}} e^{-i \omega_{i} t}+a_{s}
$$

where, we have used a mean-field approximation in which the intense control field can be treated classically and the optomechanical interaction is linearized with respect to the signal field. Here, $n_{i}(i=w, r)$ is the photon number of the writing/readout field, $\omega_{i}(i=w, r)$ is the frequency of the writing and readout field and $a_{s}$ is the annihilation operator for the signal field. The linearized Hamiltonian using Eq. (3) in Eq. (2) can be written as,

$$
H_{\text {int }} \approx-\hbar G_{i}\left(e^{-i \omega_{i} t} a_{s}^{\dagger} b+e^{i \omega_{i} t} a_{s} b^{\dagger}\right)-\hbar G_{i}\left(e^{-i \omega_{i} t} a_{s}^{\dagger} b^{\dagger}+e^{i \omega_{i} t} a_{s} b\right)
$$

where, $G_{i}=g \sqrt{n_{i}}$ is the effective optomechanical coupling rate. The first term in Eq. (4) represents the beamsplitter Hamiltonian and the second term corresponds to parametric down conversion Hamiltonian. The first term dominates when writing and readout pulses are set at one mechanical frequency below the signal frequency. In that case, the total Hamiltonian using Eq. (4) can be written as

$$
H=\hbar \omega_{s} a_{s}^{\dagger} a_{s}+\hbar \omega_{m} b^{\dagger} b-\hbar G_{i}\left(e^{-i \omega_{i} t} a_{s}^{\dagger} b+e^{i \omega_{i} t} a_{s} b^{\dagger}\right) .
$$

Now to describe the optomechanical coupling between signal field and the mechanical displacement, the master equation Eq. (1) can be unraveled in terms of the following set of Langevin equations of motion for the mean values

$$
\begin{aligned}
& \dot{\alpha}=-\left[i\left(\omega_{s}-\omega_{i}\right)+\mathcal{B}\right] \alpha+i G_{i} \beta+\sqrt{\mathcal{B} \Phi_{i n}}, \\
& \dot{\beta}=-\left[i\left(\omega_{m}-\omega_{s}+\omega_{i}\right)+\Gamma\right] \beta+i G_{i} \alpha,
\end{aligned}
$$

where, $\Gamma=\gamma_{g}+\gamma_{w}+\gamma_{s}+\gamma_{r}+\delta \Gamma$ is the mechanical damping, $\gamma_{j}=\frac{5 A_{j} \omega_{x}}{\omega_{j}}(j=t, w, s, r)$ is rate associated with the radiation damping, ${ }^{35} \delta \Gamma=12 \gamma_{f}\left(\langle N\rangle+\frac{1}{2}\right)$ is the nonlinear feedback damping and $\Phi_{i n}$ is the incident photon flux of the signal field. Note that Eqs. $(6,7)$ are derived in a frame moving with signal frequency such that $\alpha=\left\langle a_{s}\right\rangle e^{i \omega_{s} t}$ and also we define $\beta=\langle b\rangle e^{i\left(\omega_{s}-\omega_{i}\right) t}$. Further, the stochastic terms due to thermal noise and feedback backaction vanish as their expectation values are zero. It is to be pointed out that in the absence of damping, a " $\pi / 2$ pulse" perfectly maps the motional state to the optical state and optical state to the motional state thereby performing the function of the optomechanical storage and retrieval. 


\section{RESULTS}

In the optomechanical light storage, the signal pulse is stored as a mechanical excitation by a writing pulse while the readout pulse results in the retrieval of optical information from mechanical excitation. Now to discuss light storage and retrieval, we use following Gaussian pulse profiles:-

$$
G_{i}=G_{i 0} \exp \left[-\frac{\left(t-t_{i}\right)^{2}}{2 T_{i}^{2}}\right],
$$

where, $G_{i 0}, t_{i}$ and $T_{i}(i=w, s, r)$ represents the amplitude, central time and the width of the pulse, respectively.

\subsection{Optomechanical Light Storage}

To illustrate the optomechanical light storage in levitated nanoparticle, we numerically solve the coupled oscillator Eqs. $(6,7)$.
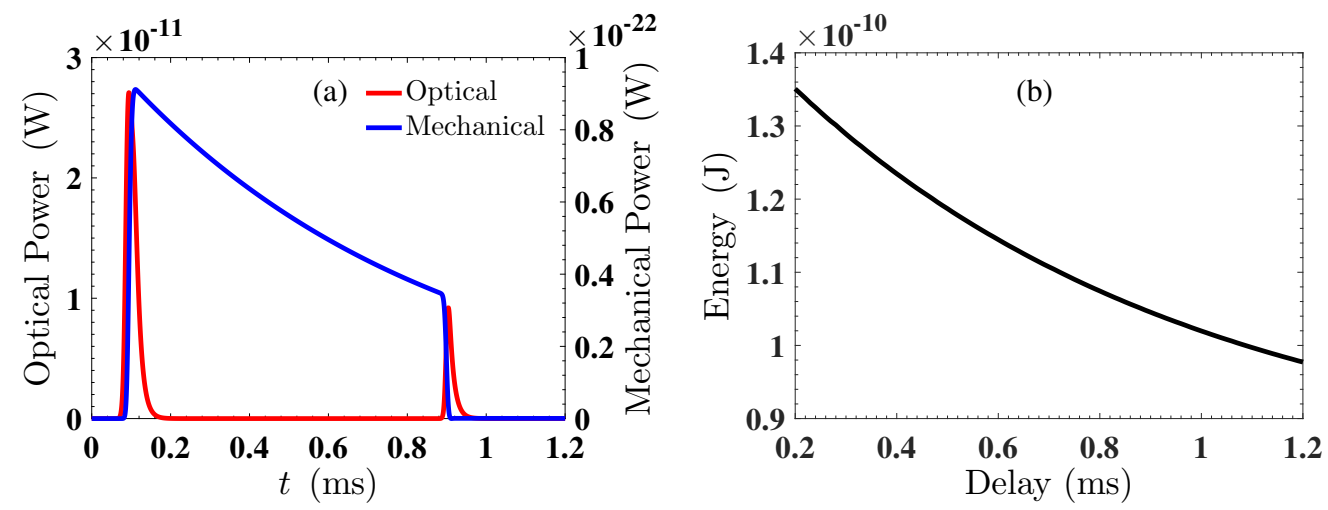

Figure 2. (a) Optical power of the storage and retrieval of the optical signal (solid red line), along with the power of the stored mechanical oscillation (solid blue line) as a function of time. (b) Retrieved pulse energy as a function of the delay between the readout and writing pulses. The parameters used in the figures are $t_{w}=t_{s}=0.09 \mathrm{~ms}, t_{r}=0.9 \mathrm{~ms}$, $T_{w}=T_{s}=T_{r}=7 \mu \mathrm{s}, G_{s 0}=4.4 \mathrm{kHz}, G_{w 0}=90 \mathrm{kHz}, G_{r 0}=110 \mathrm{kHz}, \omega_{x}=124 \mathrm{kHz}$, radius of the particle $(R)=50 \mathrm{~nm}$, mass of the nanoparticle $=1.2 \times 10^{-18} \mathrm{~kg}, \epsilon_{c}=1.133$, signal wavelength $\left(\lambda_{s}\right)=780 \mathrm{~nm}$, write wavelength $\left(\lambda_{w}\right)=1064 \mathrm{~nm}$, readout wavelength $\left(\lambda_{r}\right)=1064 \mathrm{~nm}, \Delta x=10 \mathrm{~nm}, \ell_{x}=19 \mathrm{pm}, g=0.125 \mathrm{mHz}$, Avg. write photon number $=5 \times 10^{14}, \gamma_{g}=0.0289$ $\mathrm{Hz}, \delta \Gamma=1.25 \mathrm{kHz}$, pressure $=9 \times 10^{-6}$ mbar, $\mathrm{T}=300 \mathrm{~K}, \mathcal{B}_{s}=0.3 \mathrm{~Hz}, \mathcal{B}_{w}=0.04 \mathrm{~Hz}, \mathcal{B}_{r}=0.04 \mathrm{~Hz}, \mathcal{A}_{t}=27 \mathrm{kHz}, \mathcal{A}_{s}=9 \mathrm{kHz}$, $\mathcal{A}_{w}=8 \mathrm{kHz}, \mathcal{A}_{r}=8 \mathrm{kHz}$, scaled optomechanical coupling $(\chi)=10^{-8}$ and nonlinear feedback gain $(G)=20$.

Fig. 2(a) shows the time dependence of the calculated power of the signal and the retrieved pulse, together with the power of the stored mechanical oscillation. For the storage process, a signal pulse which arrives at $t=0$ is converted into mechanical excitation by means of a writing pulse. The mechanical damping then causes the exponential decay of mechanical excitation. For the retrieval process, another readout pulse which arrives at $t=9 \mathrm{~ms}$, converts the mechanical excitation back into the retrieved optical signal, as seen from Fig. 2(a).

The optical information storage depends on the mechanical excitation which decays due to damping. Thus, the storage lifetime is limited by the mechanical decay and is determined from the dependence of the retrieved pulse energy on the delay between the writing and readout pulses, as depicted in Fig. 2(b). Clearly, the retrieved pulse energy decays exponentially with the pulse separation thereby giving a storage lifetime of $2 \mathrm{~ms}$ which is one thousand times longer than reported for cavity based optomechanical light storage. ${ }^{11}$ The longer storage lifetime in levitated optomechanics is attributed to the absence of clamping losses. Further, the storage lifetime obtained is in good agreement with mechanical linewidth $\Gamma / 2 \pi=1.25 \mathrm{kHz}$ which indicates that it is limited only by the mechanical decay of the system. The mechanical damping is mainly dominated by nonlinear feedback damping which can be controlled by changing the conditions of pressure and temperature thereby further increasing the storage lifetime. 

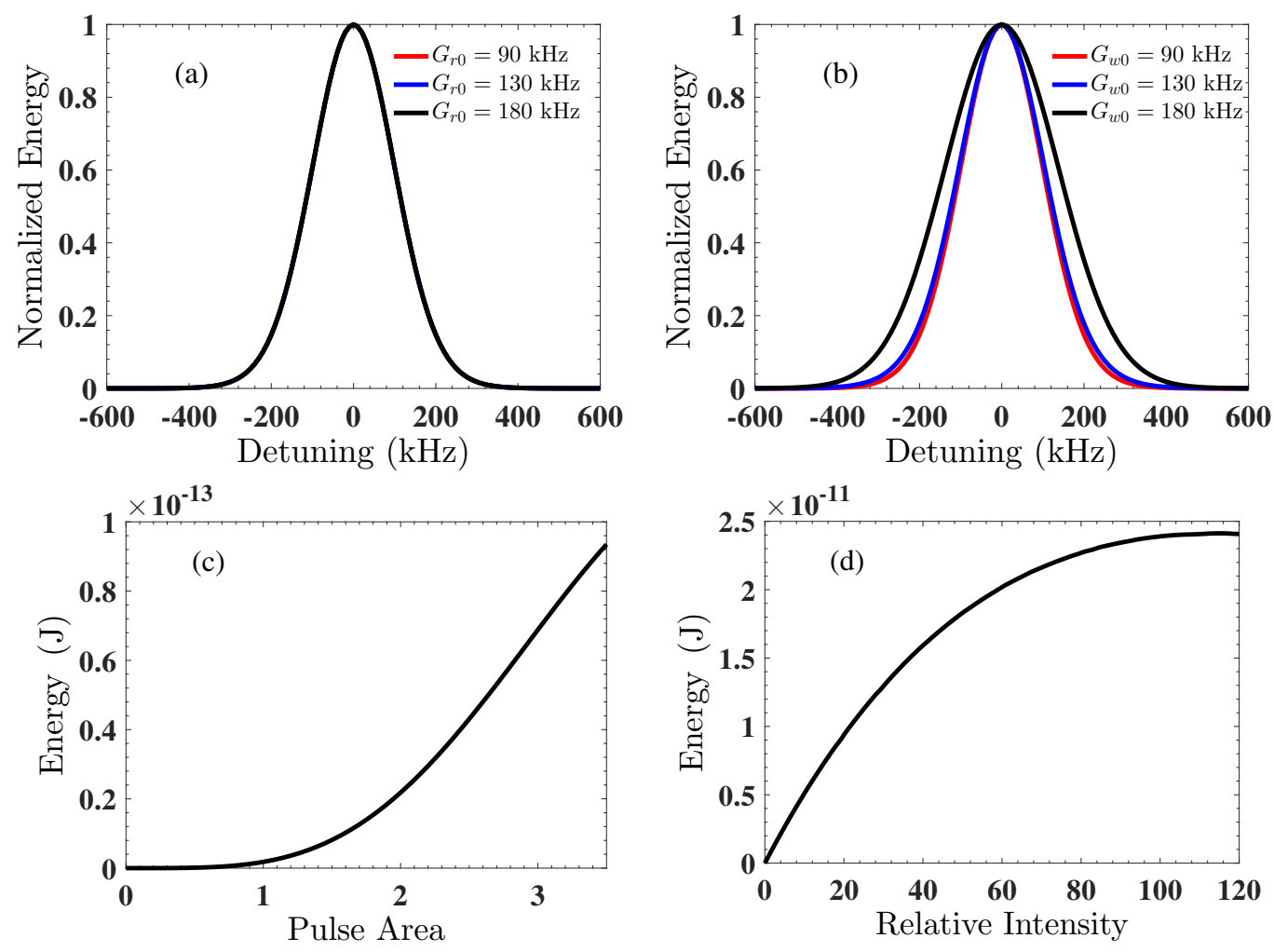

Figure 3. Normalized retrieved pulse energy as a function of detuning $\omega_{s}-\omega_{i}-\omega_{m}(i=w, r)$ for different (a) readout couplings (b) write couplings. Here energy is normalized to that retrieved at zero detuning. Retrieved pulse energy as a function of the (c) pulse area (d) relative readout intensity. Here, relative intensity of 1 corresponds to $G_{r 0}=110 \mathrm{kHz}$. Rest of the parameters are same as in Fig. 2.

The process of optomechanical light storage and retrieval is distinctly affected by writing and readout pulses. To confirm the optomechanical origin of the light storage and retrieval, we plot in Fig. 3(a), the retrieved pulse energy as a function of detuning between $\omega_{s}$ and $\omega_{i}$ for different readout intensities. The occurrence of resonance at $\omega_{s}-\omega_{i}=\omega_{x}$ validates that for light retrieval process, readout pulses is to be $\omega_{x}$ away from the signal pulse thereby satisfying the anti-Stokes resonance condition. Furthermore, the spectral lineshape of retrieved energy is independent of the power of the readout pulses, as depicted in Fig. 3(a). The light storage process, on the other hand, also requires writing pulse to be $\omega_{x}$ away from the signal which is confirmed from Fig. 3(b). A strong writing pulse gives rise to larger mechanical excitation, as a result of which broadening in the lineshape of the retrieved energy appears, as shown in Fig. 3(b).

As mentioned above, in the absence of damping, a $\pi / 2$-pulse causes a complete swap of states between optical and mechanical systems. However, in the limit $G>\Gamma$, the retrieved pulse energy rises even when the pulse area exceeds $\pi / 2$, as shown in Fig. 3(c). Further, the retrieved pulse energy is affected by the readout intensities and gets saturated for strong readout pulses (see Fig. 3(d)).

So far the above analysis is based on the conditions of low pressure $\left(\leq 10^{-5} \mathrm{mbar}\right)$. This regime of low pressure includes the case of oscillator preparation near the ground state, where a large amount of energy can be retrieved from the mechanical oscillator as depicted in 4(a). However, the storage and retrieval is drastically affected by the pressure as shown in Fig. 4(b). The increase in pressure causes the damping to increase and as a result of this the retrieved pulse energy decreases as exhibited in Fig. 4(b).

\subsection{Direction of the Retrieved Photon}

In the preceding discussion, we have described how optical information can be stored and retrieved at a later time in levitated optomechanical system. The presence of readout pulse results in the emission of a retrieved 

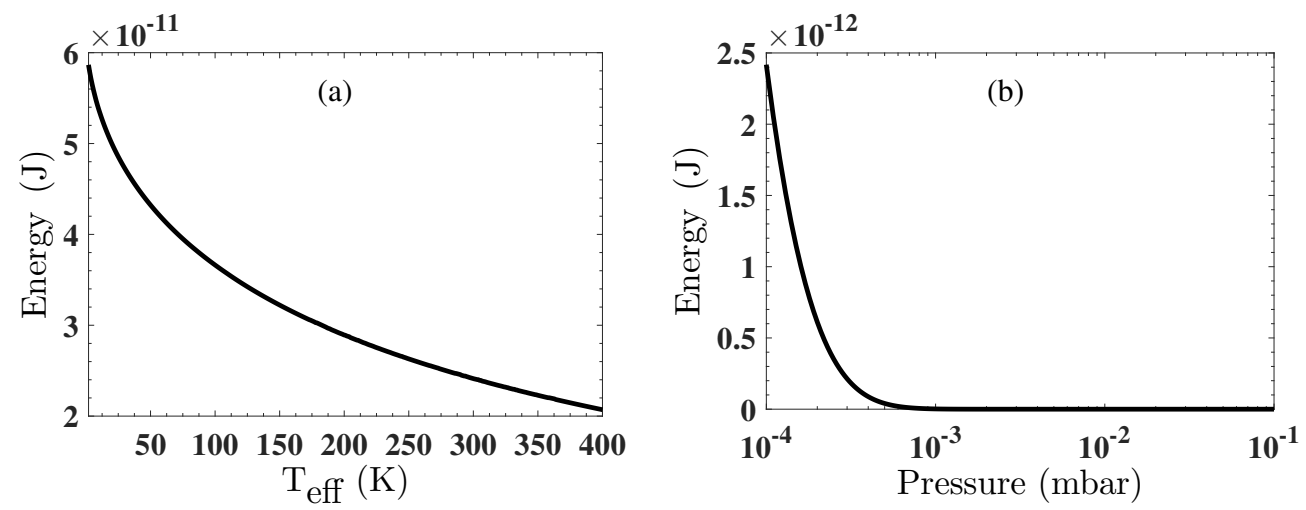

Figure 4. Retrieved pulse energy as a function of the (a) pressure and (b) temperature. Rest of the parameters are same as in Fig. 2.

photon. The direction of this photon is determined by the momentum conservation condition given by:-

$$
\vec{P}_{r}=\vec{P}_{r e t}+\Delta \vec{p}
$$

where, $\vec{P}_{r}=N_{r} \hbar \vec{k}_{r}\left(\vec{P}_{r e t}=N_{r e t} \hbar \vec{k}_{r e t}\right)$ is the momentum of the readout (retrieved) pulse with number of photons $N_{r}\left(N_{r e t}\right)$ and the wave vector $\vec{k}_{r}\left(\vec{k}_{r e t}\right)$. Also, $\Delta \vec{p}$ is the change in the momentum of the levitated nanoparticle. To find out the direction of retrieved photon, we evaluate the change in momentum of the nanoparticle which is oscillating about equilibrium position $(X=0)$ and is subjected to radiation pressure force and restoring force, as shown in Fig. 5. The momentum of the retrieved photon from Eq. (9) can be written as

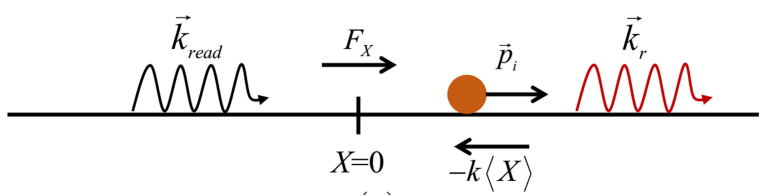

(a)

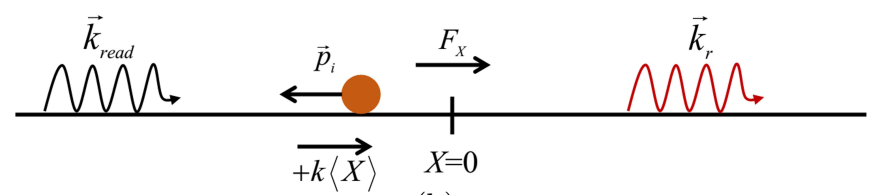

(b)

Figure 5. Schematic for the direction of retrieved photon when nanoparticle initially moves (a) away from the readout pulse (b) towards readout pulse. Here, $X=0$ is the equilibrium position about which nanoparticle oscillates, $\vec{p}_{i}$ is the initial momentum of the nanoparticle, $F_{X}$ is the radiation-pressure force and $k\langle X\rangle$ represents the restoring force.

$$
\vec{P}_{r e t}=\vec{P}_{r}-\Delta \vec{p}
$$

As is clear from Eq. (10) that if the momentum of the readout pulse dominates over the change in the momentum of the nanoparticle after impulsive interaction then the photon is retrieved in the direction of the readout pulse. Here, we demonstrate that for our system the retrieved photon moves in the direction of the readout pulse. For instance, let us consider a situation where the nanoparticle initially moves in the same direction as the readout pulse, as shown in Fig. 5(a). Under the impulsive interaction of the readout pulse, the change in momentum of the nanoparticle is determined by the radiation-pressure force $\left(F_{X}\right)$ and the restoring force $\left(F_{r e s}\right)$ due to the trap stiffness and can be written as,

$$
\Delta \vec{p}=\left(F_{X}+F_{r e s}\right) \Delta t=\left(F_{X}-k\langle X\rangle\right) \Delta t,
$$

where, $\Delta t$ is the amount of time during which impulse is imparted to the nanoparticle and $k=m \omega_{x}^{2}$ is the trap stiffness. As shown in Fig. 5(a), the direction of the restoring force is negative. Further, the position of the levitated particle can be expressed in terms of mean occupancy $\langle N\rangle$ as $\langle X\rangle=\sqrt{\frac{\langle N\rangle \hbar}{m \omega_{x}}}$. On the other hand, the 
radiation-pressure force for a situation exhibited in Fig. 5(a), points towards positive X-direction ${ }^{10}$ and by using Eq. (2) can be expressed as

$$
F_{X}=-\frac{d H_{i n t}}{d X}=\hbar \mathcal{G}_{x}|\alpha|^{2}
$$

where, $|\alpha|^{2}$ is the number of photons incident on the nanoparticle and $\mathcal{G}_{x}=\frac{2 \epsilon_{c} V \omega_{r} \Delta \omega_{r} \Delta x}{\pi^{2} w_{0}^{4} c}$. Thus, the momentum of the retrieved photon by using Eqs. $(11,12)$ can be written as,

$$
\vec{P}_{r e t}=\vec{P}_{r}-\left(F_{X}-k\langle X\rangle\right) \Delta t .
$$

Now, to figure out the direction of retrieved photon, let us use experimental parameters as used in preceding analysis to evaluate various terms on the right hand side of Eq. (13). For the parameters used in Fig. 2, for number of readout photons of $N_{r}=5 \times 10^{14}$, the momentum of the readout pulse is $\vec{P}_{r}=N_{r} \hbar \vec{k}_{r}=3.1 \times 10^{-13}$ $\mathrm{kg}^{-\mathrm{ms}^{-1}}, F_{X}=7.3 \times 10^{-14} \mathrm{~N},\langle X\rangle=1.7 \times 10^{-10} \mathrm{~m}, F_{\text {res }}=3.1 \times 10^{-18} \mathrm{~N}, N_{\text {ret }}=2 \times 10^{8}$ and $\Delta t \approx T_{r}=7 \mu \mathrm{s}$. Thus, the momentum of the retrieved photon from Eq. (13) is $\hbar \vec{k}_{r e t} \approx 1.6 \times 10^{-21} \mathrm{~kg}-\mathrm{ms}^{-1}$ and the emitted photon follows the direction of the incoming readout pulse as shown in Fig. 5(a).

However, in an opposite situation, where the nanoparticle initially oscillates towards the readout pulse, the restoring force, point towards positive x-direction, as shown in Fig. 5(b). Thus, in this case the change in momentum of the nanoparticle would be positive and for the same set of parameters as described above, the momentum of the retrieved photon from Eq. (13) would again become positive. As a result of this the photon is retrieved in the direction of the incident readout pulse as shown in Fig. 5(b).

\subsection{Conversion Efficiency}

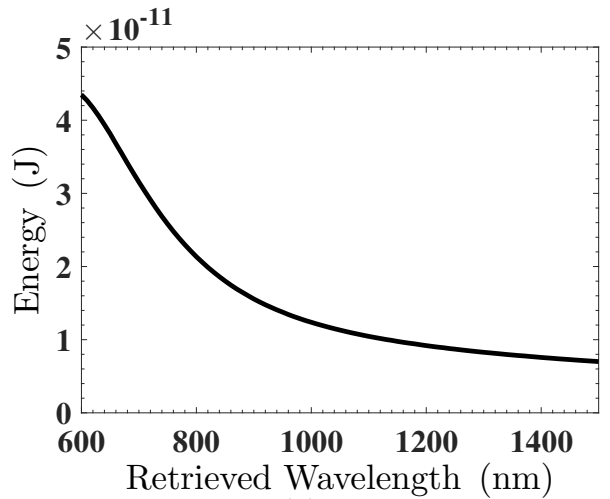

(a)

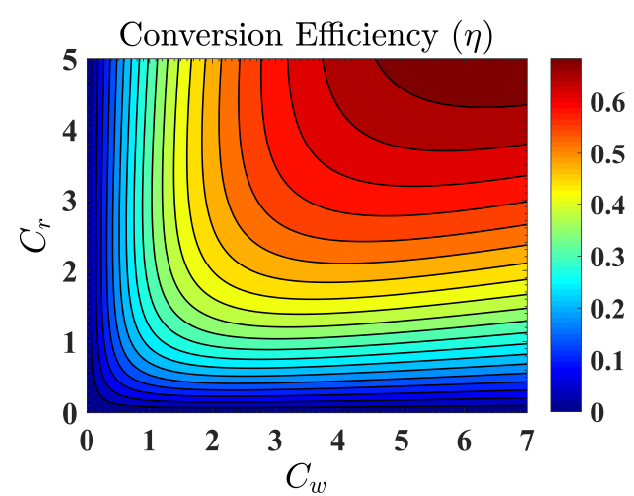

(b)

Figure 6. (a) Retrieved pulse energy as a function of the retrieved wavelength. (b) Conversion efficiency vs writing $\left(C_{w}\right)$ and readout $\left(C_{r}\right)$ cooperativities. Here signal field is converted from $780 \mathrm{~nm}$ to $1064 \mathrm{~nm}$ and the incident energy is 0.9 nJ. Other parameters are same as in Fig. 2.

In cavity based optomechanical systems, the retrieved photon needs to be resonant with the cavity. However, such a restriction is circumvented in levitated cavityless optomechanics. Here, the retrieved photon can be emitted at a wavelength different from the signal field. The wavelength of the retrieved photon is determined by energy conservation condition given by $\omega_{r}+\omega_{x}=\omega_{r e t}$. Here, $\omega_{r e t}$ is the frequency of the retrieved photon. Thus, depending on the wavelength of the readout pulse, retrieved photon can have a wavelength different from the signal field. Fig. 6(a) depicts the retrieved energy which decreases as a function of the retrieved wavelength. To further characterize the steady-state optical wavelength conversion, the optomechanical coupling $G_{w}$ maps the input signal in one optical mode to the mechanical excitation while the coupling $G_{r}$ maps the mechanical excitation to the retrieved field in another optical mode at a different frequency. In the steady state, the 
conversion efficiency, defined by the ratio of the retrieved photon flux to the input signal photon flux can be expressed as

$$
\eta=\frac{C_{w} C_{r}}{\left(1+\frac{C_{w}}{2}+\frac{C_{r}}{2}\right)^{2}}
$$

where, $C_{w}=\frac{4 G_{w}^{2}}{\mathcal{B}_{s} \Gamma}$ and $C_{r}=\frac{4 G_{r}^{2}}{\mathcal{B}_{r} \Gamma}$ are the respective optomechanical cooperativities. Note that writing and readout cooperativities depend on optomechanical coupling, optical scattering and mechanical damping which further are function of wavelength of the optical fields. ${ }^{29}$ Thus, unlike the cavity optomechanical system, the writing and readout cooperativities and hence the conversion efficiency (Eq. (14)) in levitated optomechanics can be controlled by using optical fields of any desired wavelength. Also, Eq. (14) reflects that large and equal optomechanical cooperativity for two optical modes leads to efficient wavelength conversion. Fig. 6(b) shows the photon conversion efficiency as a function of the cooperativities corresponding to $G_{w}$ and $G_{r}$. For a particular value of $C_{r}\left(C_{w}\right)$ the conversion efficiency increases with $C_{w}\left(C_{r}\right)$ and then eventually saturates thereby reflecting the requirement of impedance matching. ${ }^{15,16}$

\subsection{Amplification}

In the above discussion, we have used nonlinear feedback to cause mechanical damping and due to this the retrieved photons are obtained at lower energy than the incident signal. However, if in addition we use linear feedback heating, ${ }^{35}$ then we can get amplified retrieved photons. The inclusion of linear feedback heating ${ }^{35}$ modifies the mechanical damping term in Eqs. $(6,7)$ and can be written as $\Gamma=\gamma_{g}+\gamma_{w}+\gamma_{s}+\gamma_{r}+\delta \Gamma-\gamma_{l}$. Here, $\gamma_{l}=\chi^{2} \Phi G_{h}$ represents the linear feedback heating and $G_{h}$ is the corresponding gain. The linear feedback heating causes a decrease in the mechanical damping. Thus, when the readout pulse comes, then it retrieves more energy from the mechanical excitation thereby causing amplification of the retrieved photon. Fig. 7 (a) depicts such amplification of the retrieved photons by the application of linear feedback heating of $\gamma_{l}=1.17 \mathrm{kHz}$.
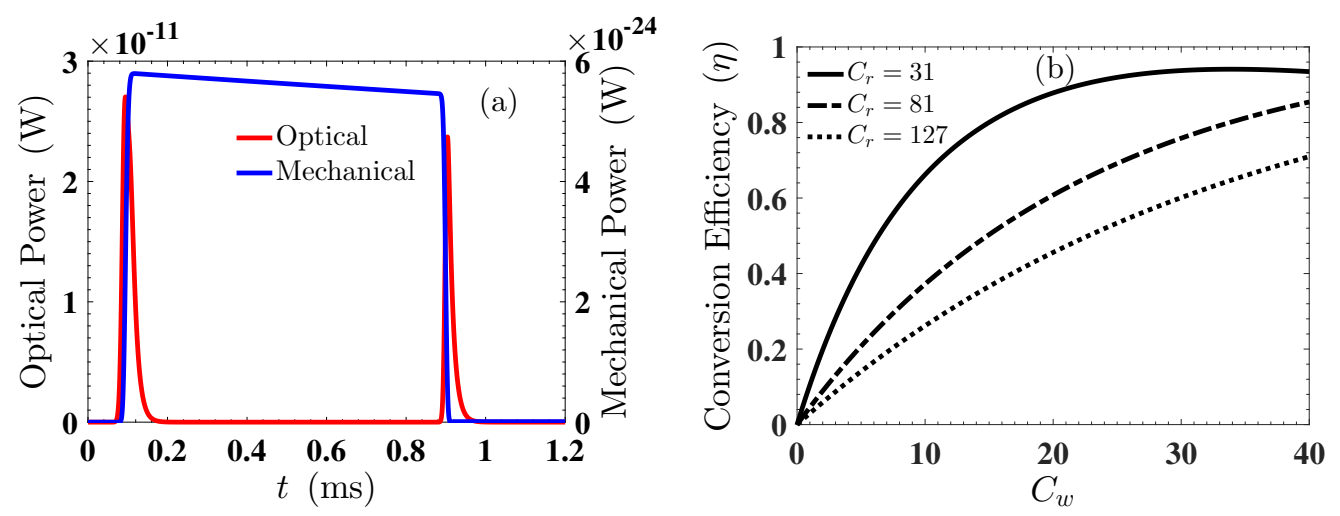

Figure 7. (a) Amplification in the light storage and retrieval process. Optical power of the storage and retrieval of the optical signal (solid red line), along with the power of the stored mechanical oscillation (solid blue line) as a function of time. (b) Conversion efficiency as a function of the writing cooperativity $\left(C_{w}\right)$ for different values of readout cooperativity $\left(C_{r}\right)$. Here, $\gamma_{l}=1.17 \mathrm{kHz}, G_{h}=95$ and other parameters are same as in Fig. 2.

The conversion efficiency, as shown in Fig. 7(b) for the case of amplification first increases with input signal cooperativity and then saturates implying the impedance matching.

\section{CONCLUSION}

In conclusion, we have described an optical memory protocol using a single nanoparticle levitated in an optical dipole trap. This is achieved by controlling the coupling between mechanical displacement and signal field by using a writing and readout pulse at one mechanical frequency below the signal field. It is shown that during 
the write pulse, a signal pulse at a given wavelength is stored as a mechanical excitation of the nanoparticle oscillation. Then a readout pulse at later time can retrieve the stored optical information from the mechanical oscillator at a desired wavelength. We have demonstrated that due to the absence of clamping losses a longer storage lifetime can be achieved in the levitated optomechanical light storage. Further, we have illustrated that our protocol can be used for wavelength conversion and shows a saturation in the conversion efficiency. We also have described that the presence of positive linear feedback causes the amplification of the retrieved photon. Moreover, the light storage with levitated optomechanics can be used to explore the possibility of quantum memories for photonic states. Our prototype for light storage works at room temperature and backaction due to nonlinear feedback damping is neglected for classical applications which otherwise could restrict this technique for the storage of single photon at a quantum level. However, with the recent progress towards cooling a levitated mechanical oscillator to its quantum ground state, ${ }^{27-29,34}$ it would be interesting to store single photons in such systems for quantum memory.

\section{ACKNOWLEDGMENTS}

This research was funded by the Office of Naval Research (ONR) under Award No. N00014-14-1-0803.

\section{REFERENCES}

[1] Briegel, H.-J., Dür, W., Cirac, J. I., and Zoller, P., "Quantum repeaters: The role of imperfect local operations in quantum communication," Phys. Rev. Lett. 81, 5932-5935 (Dec 1998).

[2] Fleischhauer, M. and Lukin, M. D., "Dark-state polaritons in electromagnetically induced transparency," Phys. Rev. Lett. 84, 5094-5097 (May 2000).

[3] Liu, C., Dutton, Z., Behroozi, C. H., and Hau, L. V., "Observation of coherent optical information storage in an atomic medium using halted light pulses," Nature 409, 490-493 (Jan 2001).

[4] Zhao, R., Dudin, Y. O., Jenkins, S. D., Campbell, C. J., Matsukevich, D. N., Kennedy, T. A. B., and Kuzmich, A., "Long-lived quantum memory," Nature Physics 5, 100-104 (Dec 2008).

[5] Lukin, M. D., "Colloquium: Trapping and manipulating photon states in atomic ensembles," Rev. Mod. Phys. 75, 457-472 (Apr 2003).

[6] Grezes, C., Julsgaard, B., Kubo, Y., Ma, W. L., Stern, M., Bienfait, A., Nakamura, K., Isoya, J., Onoda, S., Ohshima, T., Jacques, V., Vion, D., Esteve, D., Liu, R. B., Mølmer, K., and Bertet, P., "Storage and retrieval of microwave fields at the single-photon level in a spin ensemble," Phys. Rev. A 92, 020301 (Aug 2015).

[7] Krimer, D. O., Zens, M., Putz, S., and Rotter, S., "Sustained photon pulse revivals from inhomogeneously broadened spin ensembles," Laser \& Photonics Reviews 10, 1023-1030 (Oct 2016).

[8] de Riedmatten, H., Afzelius, M., Staudt, M. U., Simon, C., and Gisin, N., "A solid-state light-matter interface at the single-photon level," Nature 456, 773-777 (Dec 2008).

[9] Lauritzen, B., Mináŕ, J., de Riedmatten, H., Afzelius, M., Sangouard, N., Simon, C., and Gisin, N., "Telecommunication-wavelength solid-state memory at the single photon level," Phys. Rev. Lett. 104, 080502 (Feb 2010).

[10] Aspelmeyer, M., Kippenberg, T. J., and Marquardt, F., "Cavity optomechanics," Rev. Mod. Phys. 86, 1391-1452 (Dec 2014).

[11] Fiore, V., Yang, Y., Kuzyk, M. C., Barbour, R., Tian, L., and Wang, H., "Storing optical information as a mechanical excitation in a silica optomechanical resonator," Phys. Rev. Lett. 107, 133601 (Sep 2011).

[12] Fiore, V., Dong, C., Kuzyk, M. C., and Wang, H., "Optomechanical light storage in a silica microresonator," Phys. Rev. A 87, 023812 (Feb 2013).

[13] Tian, L. and Wang, H., "Optical wavelength conversion of quantum states with optomechanics," Phys. Rev. A 82, 053806 (Nov 2010).

[14] Stannigel, K., Rabl, P., Sørensen, A. S., Zoller, P., and Lukin, M. D., "Optomechanical transducers for long-distance quantum communication," Phys. Rev. Lett. 105, 220501 (Nov 2010).

[15] Safavi-Naeini, A. H. and Painter, O., "Proposal for an optomechanical traveling wave phononphoton translator," New Journal of Physics 13, 013017 (Jan 2011). 
[16] Dong, C., Fiore, V., Kuzyk, M. C., Tian, L., and Wang, H., "Optical wavelength conversion via optomechanical coupling in a silica resonator," Annalen der Physik 527, 100-106 (Sep 2015).

[17] O'Connell, A. D., Hofheinz, M., Ansmann, M., Bialczak, R. C., Lenander, M., Lucero, E., Neeley, M., Sank, D., Wang, H., Weides, M., Wenner, J., Martinis, J. M., and Cleland, A. N., "Quantum ground state and single-phonon control of a mechanical resonator," Nature 464, 697-703 (Mar 2010).

[18] Chan, J., Alegre, T. P. M., Safavi-Naeini, A. H., Hill, J. T., Krause, A., Gröblacher, S., Aspelmeyer, M., and Painter, O., "Laser cooling of a nanomechanical oscillator into its quantum ground state," Nature 478, 89-92 (Oct 2011).

[19] Gröblacher, S., Hammerer, K., Vanner, M. R., and Aspelmeyer, M., "Observation of strong coupling between a micromechanical resonator and an optical cavity field," Nature 460, 724-727 (Aug 2009).

[20] Agarwal, G. S. and Huang, S., "Electromagnetically induced transparency in mechanical effects of light," Phys. Rev. A 81, 041803 (Apr 2010).

[21] Weis, S., Rivière, R., Deléglise, S., Gavartin, E., Arcizet, O., Schliesser, A., and Kippenberg, T. J., "Optomechanically induced transparency," Science 330, 1520-1523 (Dec 2010).

[22] Safavi-Naeini, A. H., Alegre, T. P. M., Chan, J., Eichenfield, M., Winger, M., Lin, Q., Hill, J. T., Chang, D. E., and Painter, O., "Electromagnetically induced transparency and slow light with optomechanics," Nature 472, 69-73 (Mar 2011).

[23] Chang, D. E., Regal, C. A., Papp, S. B., Wilson, D. J., Ye, J., Painter, O., Kimble, H. J., and Zoller, P., "Cavity opto-mechanics using an optically levitated nanosphere," Proceedings of the National Academy of Sciences 107, 1005-1010 (Jan 2010).

[24] Neukirch, L. P. and Vamivakas, A. N., "Nano-optomechanics with optically levitated nanoparticles," Contemporary Physics 56, 48-62 (Sep 2015).

[25] Romero-Isart, O., Clemente, L., Navau, C., Sanchez, A., and Cirac, J. I., "Quantum magnetomechanics with levitating superconducting microspheres," Phys. Rev. Lett. 109, 147205 (Oct 2012).

[26] Cirio, M., Brennen, G. K., and Twamley, J., "Quantum magnetomechanics: Ultrahigh- $q$-levitated mechanical oscillators," Phys. Rev. Lett. 109, 147206 (Oct 2012).

[27] Li, T., Kheifets, S., and Raizen, M. G., "Millikelvin cooling of an optically trapped microsphere in vacuum," Nature Physics 7, 527-530 (Mar 2011).

[28] Arita, Y., Mazilu, M., and Dholakia, K., "Laser-induced rotation and cooling of a trapped microgyroscope in vacuum," Nature Communications 4, 2374 (Aug 2013).

[29] Rodenburg, B., Neukirch, L. P., Vamivakas, A. N., and Bhattacharya, M., "Quantum model of cooling and force sensing with an optically trapped nanoparticle," Optica 3, 318-323 (Mar 2016).

[30] Romero-Isart, O., Juan, M. L., Quidant, R., and Cirac, J. I., "Toward quantum superposition of living organisms," New Journal of Physics 12, 033015 (Mar 2010).

[31] Moore, D. C., Rider, A. D., and Gratta, G., "Search for millicharged particles using optically levitated microspheres," Phys. Rev. Lett. 113, 251801 (Dec 2014).

[32] Ranjit, G., Atherton, D. P., Stutz, J. H., Cunningham, M., and Geraci, A. A., "Attonewton force detection using microspheres in a dual-beam optical trap in high vacuum," Phys. Rev. A 91, 051805 (May 2015).

[33] Kumar, P. and Bhattacharya, M., "Magnetometry via spin-mechanical coupling in levitated optomechanics," Opt. Express 25, 19568-19582 (Aug 2017).

[34] Gieseler, J., Deutsch, B., Quidant, R., and Novotny, L., "Subkelvin parametric feedback cooling of a lasertrapped nanoparticle," Phys. Rev. Lett. 109, 103603 (Sep 2012).

[35] Jain, V., Gieseler, J., Moritz, C., Dellago, C., Quidant, R., and Novotny, L., "Direct measurement of photon recoil from a levitated nanoparticle," Phys. Rev. Lett. 116, 243601 (Jun 2016).

[36] Ge, W., Rodenburg, B., and Bhattacharya, M., "Feedback-induced bistability of an optically levitated nanoparticle: A Fokker-Planck treatment," Phys. Rev. A 94, 023808 (Aug 2016). 